\title{
36 Kurzdarmsyndrom und abdominelle Fisteln
}

Manuel Braun, Alfred Königsrainer

\subsection{Kapitelzusammenfassung}

Ein Kurzdarmsyndrom unterschiedlichster Ursachen liegt immer dann vor, wenn durch Verlust eine zu geringe intestinale Oberfläche vorliegt und dadurch eine suffiziente enterale Ernährung nicht mehr möglich ist. Erweiterte Darmresektionen bei intestinalen sowie vaskulären Erkrankungen und Bauchtraumata sind dabei die häufigsten Ursachen des Darmverlustes beim Erwachsenen. Ebenso können Motilitätsstörungen, ausgedehnte Desmoide des Abdomens und Fisteln zu einem funktionellen Kurzdarmsyndrom führen. Das intestinale Versagen beschreibt ein multifaktorielles Syndrom, dem beinahe immer ein Kurzdarmsyndrom vorausgeht. Abdominelle Fisteln, insbesondere die sogenannten high-output Fisteln können ein intestinales Versagen im Sinne eines funktionellen oder „echten“ Kurzdarmsyndroms verursachen und entstehen meist als Komplikation nach Baucheingriffen oder im Rahmen eines Morbus Crohn.

Im Vordergrund des therapeutischen Management ist die Aufrechthaltung der Nutrition (parenteral oder enteral), Förderung der Adaptation und Rehabilitation des Darmes und Vermeidung von sekundären Komplikationen wie zum Beispiel Sepsis, Wundinfektion, Elektrolytstörungen oder Leberschäden durch langanhaltende parenterale Ernährung (IFALD, intestinal failure associated liver disease).

Das folgende Kapitel hat zum Ziel einen Überblick über Ursachen des Kurzdarmsyndroms und abdomineller Fisteln zu geben. Nicht zuletzt wird auch die stadiengerechte Therapie von konservativ über invasiv und nicht zuletzt auch die Darmtransplantation in Kürze erläutert.

\subsection{Definition}

\subsubsection{Kurzdarmsyndrom}

Das Kurzdarmsyndrom ist weitgehend immer Voraussetzung eines intestinalen Versagens. Die 2014 publizierte S3-Leitlinie der Deutschen Gesellschaft für Ernährungsmedizin definiert intestinales Versagen als Unfähigkeit die Protein-, Energie-, Flüssigkeits- und Mikronährstoffbilanz aufrechtzuhalten.

Es tritt auf, wenn bei optimal adaptiertem Darm, worunter ein Zuwachs an Resorptionsoberfläche der Darmzotten zu verstehen ist, weniger als $60 \mathrm{~cm}$ Dünndarm inclusive Dickdarm, oder $100 \mathrm{~cm}$ Dünndarm ohne Dickdarm vorliegen. Bei noch nicht adaptiertem Darm kann vereinfacht dargestellt bei residueller Darmlänge von weniger als $200 \mathrm{~cm}$ von einem apparenten Kurzdarmsyndrom ausgegangen werden. 


\subsubsection{Abdominelle Fisteln}

Eine Fistel ist eine pathologische Verbindung zwischen zwei epithelialisierten Oberflächen. Eine enterokutane Fistel ist eine Verbindung zwischen Darm und der Haut. Enteroatmosphärische Fisteln sind eine Untergruppe der enterokutanen Fisteln, bei denen die Darmmukosa von außen frei sichtbar ist, ohne darüberliegendes Gewebe wie z. B. Bauchmuskulatur.

Sie können mit der Trias Sepsis, Malnutrition und Flüssigkeits-/Elektrolytstörungen vergesellschaftet sein.

Klinische Apparenz (,high-output“) erlangen Fisteln in Bezug auf Kurzdarmsymptomatik bei Fördermengen ab 1500-2000 ml pro Tag.

\section{3 Ätiologie}

\subsubsection{Kurzdarmsyndrom}

Das Kurzdarmsyndrom ist mit knapp über $60 \%$ die häufigste Ursache des chronischen intestinalen Versagens. Es entsteht entweder durch eine einzelne weitläufige Resektion von Darm oder durch wiederholte Resektionen. Die Ursachen hierfür variieren je nach Altersgruppe der Patienten. Bei Säuglingen steht die nekrotisierende Enterokolitis mit 40-50 \% aller Fälle an erster Stelle, gefolgt von Darmatresie, Gastroschisis und Volvulus. Bei älteren Kindern sind Traumata, Operationskomplikationen und Malignome häufige Ursachen.

Bei Erwachsenen entsteht das Kurzdarmsyndrom am häufigsten durch postoperative Komplikationen (36\%) wie z. B. Fisteln, Volvulus, innere Hernien oder Ileus. Ebenfalls häufige Ursachen mit bis zu 20 \% stellen tumorbedingte massive Resektionen (z. B. stromale Tumoren) mit oder ohne subsequente Bestrahlung und Strahlenenteritis dar. Mesenterialgefäßverschlüsse durch Thrombosen oder Embolien stellen nebst kompliziertem Morbus Crohn aufgrund der oftmals notwendigen aggressiven oder repetitiven Resektionen ebenfalls häufige Ätiologien eines Kurzdarmsyndroms dar.

Je nach Ursache kann es sich dann um ein akutes, selbstlimitierendes Darmversagen/Kurzdarmsyndrom oder um ein chronisches womöglich irreversibles Darmversagen/Kurzdarmsyndrom handeln.

In Tab.36.1 sind die häufigsten Ursachen eines Kurzdarmsyndroms nach Altersgruppe der Patienten aufgeführt. 
Tab. 36.1: Häufigste Ursachen des Kurzdarmsyndroms nach Altersgruppe der Patienten.

\begin{tabular}{ll}
\hline Säuglinge & - nekrotisierende Enterokolitis \\
& - Darmatresie \\
& - Gastroschisis \\
& - Volvulus \\
& - angeborene Störungen \\
\hline Kinder & - Traumata \\
& - postoperative Komplikationen \\
& - Malignome \\
& - Motilitätsstörungen \\
\hline Erwachsene & - postoperative Komplikationen \\
& - Malignome/ post Radiatio \\
& - Mesenterialgefäßverschlüsse \\
& - Morbus Crohn \\
& - Traumata \\
& - Volvulus \\
& - Motilitätsstörungen \\
\hline
\end{tabular}

\subsubsection{Fisteln}

Die Entstehung von Fisteln basiert in der Regel entweder auf einer Darmerkrankung die sich nach extraluminal ausweitet, einer extraluminalen Pathologie welche den Darm befällt, Abdominaltraumata oder dem Auftreten einer Anastomoseninsuffizienz. 75-85\% aller Fisteln treten dabei in Zusammenhand mit einer stattgehabten Operation als Komplikation dieser auf und sind somit iatrogen. Die Operationen welche mit Fisteln gehäuft vergesellschaftet sind, sind Tumorresektionen, Operationen bei chronisch entzündlichen Darmerkrankungen, Adhäsiolyse, Pankreasresektionen oder die Versorgung von Bauchwandhernien. Hierbei sind das mögliche Auftreten von Anastomoseninsuffizienzen oder versehentliche intraoperative Darmverletzungen ausschlaggebend. Die Implantation von Netzmaterial in der Hernienchirurgie erhöht ebenfalls das Risiko für Fistelformation. Die übrigen 15-25\% treten unabhängig eines Eingriffes aufgrund der zugrundeliegenden Pathologie auf. Die häufigsten Ursachen sind hierbei chronisch entzündliche Darmerkrankungen (insbesondere Morbus Crohn), Strahlenenteritis, komplizierte Divertikelkrankheit, Pankreatitis, Appendizitis, perforierte Ulzera, Tumore oder Traumata.

Fisteln repräsentieren mit $50 \%$ das Gros der Patienten mit akutem intestinalem Versagen und wiederum $50 \%$ dieser Patienten hat einen komplizierten Morbus Crohn.

In Tab. 36.2 ist eine Übersicht der häufigsten Ursachen von Fisteln nach Ätiologie und Häufigkeit aufgeführt. 
Tab. 36.2: Ursachen von abdominellen Fisteln nach Ätiologie und Häufigkeit dargestellt.

\begin{tabular}{ll}
\hline $\begin{array}{l}\text { iatrogene Fisteln } \\
(75-85 \%)\end{array}$ & - postoperativ im Sinne einer Komplikation \\
\hline spontane Fisteln & - Morbus Crohn \\
$(15-25 \%)$ & - Strahlenenteritis \\
& - komplizierte Divertikelkrankheit \\
& - perforierte Ulzera \\
& - Tumorperforationen \\
& - Pankreatitis \\
\hline
\end{tabular}

\subsection{Klassifikation}

\subsubsection{Kurzdarmsyndrom/intestinales Versagen}

Das intestinale Versagen wird gemeinhin entweder in zwei oder drei Typen klassifiziert. In der bivalenten Klassifikation unterscheidet man zwischen akutem, oft transientem Darmversagen (Typ 1) und chronischem, oft persistierendem Darmversagen (Typ 2).

Die S3-Leitlinie der Deutschen Gesellschaft für Ernährungsmedizin klassifiziert Typ 1-3. Typ 1 ist dabei akut, kurz und selbstlimitierend. Typ 2 bezeichnet einen prolongierten akuten Zustand über mehrere Wochen bis Monate mit der Notwendigkeit einer interdisziplinären Versorgung mitunter mit parenteraler Ernährung. Typ 3 betrifft jene Patienten, die über Jahre hinweg oder irreversibel von parenteraler Ernährung abhängig sind, jedoch einen metabolisch und klinisch stabilen Zustand haben.

\subsubsection{Fisteln}

Fisteln können nach Anatomie, Physiologie oder Ätiologie klassifiziert werden. Die anatomische Klassifikation richtet sich dabei nach dem Ursprung der Fistel, z. B. enterokutane Fistel oder gastrokutane Fistel etc. Für die ätiologische Klassifikation siehe oben. Die physiologische Klassifikation richtet sich nach dem Fördervolumen. So unterscheidet man in:

- Low-output Fistel $\leq 200 \mathrm{ml} / \mathrm{d}$

- $\quad$ Moderate-output Fistel $=200-500 \mathrm{ml} / \mathrm{d}$

- High-output Fistel $\geq 500 \mathrm{ml} / \mathrm{d}$

Je höher die Fördermenge der Fistel ist, desto gravierender sind die klinischen Implikationen und die Heilungstendenz. 


\subsection{Symptomatik}

Die Problematik welche insbesondere aus einer high-output Fistel zutage treten kann, kann mit der o. g. Trias von Sepsis, Malnutrition und Flüssigkeits-/Elektrolytstörungen zusammengefasst werden. Ferner können entlang des Fisteltraktes Abszesse oder Wunden mit chronischen Hautschäden entstehen.

\subsection{Diagnostik}

Ein grundlegendes Monitoring der Vitalparameter und Laborchemie um eine Dehydration, Elektrolytentgleisung, Ernährungszustandsverschlechterung oder septische Entwicklung zu erkennen ist Grundvoraussetzung. Hierbei sollten regelmäßig Blut-, Urin- und Stuhlproben gewonnen und untersucht werden. Insbesondere das Screening auf Nährstoff- und Vitaminmangel sollten nicht vernachlässigt werden.

Um die Versorgung der Fisteln verbessern zu können oder zur Statuserhebung nachdem sich der Patient klinisch stabilisiert hat, haben sich diverse radiologische Untersuchungsmodalitäten bewährt. Mittels Fistulogramm kann nach Intubation und Applikation eines Kontrastmittels ein großer Informationsgewinn erzielt werden. Hiermit können Länge, Ursprung, Traktverlauf, distale Stenosen am Darm o. Ä. ermittelt werden. Zur Abszesslokalisation bzw. -detektion oder Bewertung der Fistelumgebung oder des Darmes kann eine CT-Bildgebung oder MR-Bildgebung gewonnen werden. Bei Fisteln im oberen Gastrointestinaltrakt kann eine Endoskopie möglicherweise wichtige Erkenntnisse oder Therapieoptionen bieten.

\subsection{Therapie konservativ}

Je nach Schweregrad der Ausprägung der Fistel und dem ggf. begleitendem Darmversagen können unterschiedliche Konzepte zum Einsatz kommen. Die Therapie bedarf jedoch sowohl in der konservativen als auch chirurgischen Vorgehensweise das enge Zusammenspiel mehrerer Experten. Hierunter sind Heilungschancen von bis zu 85\% erzielbar. Allein durch eine suffiziente konservative Therapie können ca. ein Drittel aller Fisteln spontan abheilen.

Ziel der Therapie ist dabei hinsichtlich des Kurzdarmsyndroms die Etablierung eines ausgeglichenen Energie- und Flüssigkeitshaushaltes unter Berücksichtigung der Lebensumstände und Anforderungen an Mikro- und Makronährstoffen. In Bezug auf das Fistelleiden können vier wichtige Therapiekriterien genannt werden:

- Flüssigkeitsausgleich/Nutrition

- Drainage möglicher Abszesse/Sepsiskontrolle

- Kontrolle der Ausfuhr

- Hautschutz/Wundversorgung 
Eine erhebliche Morbidität und Mortalität entsteht auf lange Sicht gesehen durch das Risiko von rezidivierender Sepsis, Rezidivfisteln sowie Leberversagen. Daher zielt die Therapie neben den o. g. Punkten auch im Besonderen auf die Vermeidung von Komplikationen.

\subsubsection{Flüssigkeitsausgleich/Nutrition}

Insbesondere bei hohen Dünndarmfisteln kann es $\mathrm{zu}$ einer massiven high-output Situation kommen, welche Flüssigkeitsverluste von über $4 \mathrm{~L} / 24 \mathrm{~h}$ verursachen kann. Dabei ist das Auftreten einer Hypokalämie die häufigste Elektrolytentgleisung und diese sollte aggressiv ausgeglichen werden. In der Regel können die hochvolumigen Flüssigkeitsverluste aus dem oberen Gastrointestinaltrakt nur mithilfe von intravenöser Flüssigkeitstherapie mittels Vollelektrolytlösung ersetzt werden. Sind die Flüssigkeitsverluste oral kompensierbar, so kann bei rezidivierenden Elektrolytentgleisungen auf orale Rehydratationslösungen zurückgegriffen werden. Generell sollten hypotonische Getränke wie z. B. reines Wasser nur limitiert eingenommen werden, da diese eine Diffusion von Wasser und Natrium ins Darmlumen bedingen und dadurch den Flüssigkeitsverlust erhöhen können.

Bezüglich der Ernährung sollte falls möglich die enterale Ernährung favorisiert werden. Dies begünstigt die Adaptation des Darmes, also die Erhöhung der resorptiven Kapazität durch Wachstum der Darmzotten und scheint das Risiko für Thrombosen und Infektionen zu verringern. Eine hochkalorische und ballaststoffarme Ernährung ist dabei vorzuziehen, da so eine möglichst langsame Passage und somit längere Resorptionszeit erzielt werden. Bei intaktem Kolon können auch über das Kolon mittelkettige Fettsäuren resorbiert werden. Diese stellen somit insbesondere zu Beginn der Adaptationsphase die Fettquelle der Wahl dar. Auch über die Fistel kann ernährt werden, sofern der abführende Darm kanuliert werden kann und mindestens $75 \mathrm{~cm}$ aboraler Darm zur Verfügung stehen.

Sollte eine enterale Ernährung nicht möglich sein oder ausreichen, so stellt die parenterale Ernährung mittlerweile eine etablierte Option dar. Zur genauen Berechnung der erforderlichen Makronährstoffe und Mikronährstoffe stehen mittlerweile hochspezialisierte Ernährungsexperten zur Verfügung. Im Allgemeinen benötigt ein Patient 25-32 kcal/Tag bei einer Eiweißzufuhr von 1,5 g/Kg/KG. Das Erzielen eines guten Ernährungszustandes hat hohe Bedeutung, da dies die Morbidität senkt und die Heilungschancen signifikant verbessert. 


\subsubsection{Drainage möglicher Abszesse/Sepsiskontrolle}

Sepsis und intraabdominelle Abszesse sind eine ernstzunehmende Komplikation in dieser Patientenpopulation. Mittels Bildgebung können eventuelle Verhaltformationen detektiert werden und heutzutage in der Regel mittels CT-gesteuerter Drainage behandelt werden. Bei unzureichender Drainage kann entlang der einliegenden Drainage geschnitten und dickere Drainagen appliziert werden. Eine Re-Laparotomie sollte möglichst in den ersten 6 Monaten vermieden werden.

\subsubsection{Kontrolle der Ausfuhr}

Eine wirkungsvolle Ausfuhrkontrolle wirkt sich in vielerlei Hinsicht positiv aus. Zum einen verringert sich dadurch der zum Teil sehr erhebliche Flüssigkeitsverlust, zum anderen kann hiermit die enterale Nutrition verbessert werden, da eine längere Transitzeit die Resorptionszeit erhöht. Ein geringerer Fluss über die Fistel stellt nicht zuletzt für den Patienten eine pflegerisch bessere Situation dar.

Im Allgemeinen kommen hierfür Antidiarrhoika zum Einsatz, wie z. B. hochdosiertes Loperamid oder Tinctura opii. Aufgrund des z. T. fehlenden enterohepatischen Kreislaufs muss hierbei mit sehr hoher Dosierung gearbeitet werden. Insgesamt konnte mit o. g. Mitteln eine Reduktion des Wasser- und Salzverlustes um 20-30\% gezeigt werden. Eine Reduktion der Magensekretion kann mittels Protonenpumpeninhibitoren oder H2-Blockern etabliert werden. Dabei scheinen H2-Blocker die Magensekretion wirkungsvoller zu verringern. Trotz reduziertem Flüssigkeitsverlust scheinen die Magensäureblocker keinen positiven Einfluss auf die Resorption auszuüben.

Clonidin subkutan konnte bei high-output Stomas und Fisteln eine wirksame Reduktion der Fördermenge zeigen und ist somit als Therapiezusatz denkbar, sofern die Kreislaufsituation das zulässt.

Cholestyramin kann bei chologener Diarrhoe in Erwägung gezogen werden. In Studien konnte hiermit eine verbesserte Resorption von Nahrungsfetten und Kalzium gezeigt werden.

Antisekretorisch können ebenfalls die synthetischen Abkömmlinge des Somatostatins angewendet werden. Somatostatin selbst hat aufgrund seiner Halbwertszeit von 1-3 Minuten nur eine begrenzte klinische Anwendbarkeit. Mittels dem Analogon Octreotid (Halbwertszeit 2h) konnte in Studien eine Output-Reduktion von 40-93\% demonstriert werden und somit indirekt auch eine deutlich verkürzte Verheildauer und erhöhte Tendenz zur Spontanheilung erzielt werden.

Teduglutide ist ein Analogon des Glucagon-Like Peptide 2 und gilt als intestinotrophes Medikament. Es stimuliert das Wachstum der Mukosazellen im Dünndarm, insbesondere im Jejunum und kann somit die Resorptionsfläche des Darmes erhöhen. Bei Langzeitanwendung von Teduglutide sind engmaschige Koloskopien von Nöten, da eine vermehrte Adenomentstehung damit vergesellschaftet ist. 


\subsubsection{Hautschutz/Wundversorgung}

Die Haut um die Fistel herum ist mitunter hohen Belastungen ausgesetzt, wie z. B. dauerhafte Nässe oder irritierende Substanzen im Sekret. Bei enteroatmosphärischen Fisteln besteht meist auch eine riesige Wumdumgebung. Gerade hierfür ist das Hinzuziehen von geschulten Wund- und Stomafachpflegenden elementar. Die Sicherheit und Anwendbarkeit von Vakuumverbandssystemen bei enteroatmosphärischen Fisteln ist noch nicht abschließend geklärt. Zum einen kann der verbesserte Sekretabtransport zu einer verbesserten Wundheilung führen, jedoch scheint ein leicht erhöhtes Risiko, wenn auch nicht signifikant, für die Entstehung weiterer Fisteln unter dem dauerhaften Sog zu bestehen.

\subsection{Therapie operativ}

Im Hinblick auf die Kurzdarmsymptomatik ist in der Regel die primäre chirurgische Intervention die Etablierung eines langfristigen zentralvenösen Zuganges, um die Flüssigkeits- und Ernährungstherapie sicherzustellen. Hierbei sollten Venen der oberen Körperhälfte aufgrund der deutlich geringeren Infektions- und Thrombosegefahr und nicht zuletzt auch wegen des Patientenkomforts und der geringeren Neigung zu Okklusion bevorzugt werden. Bezüglich der Wahl der Katheterart stehen zur Langzeitversorgung das Portsystem und der Hickmankatheter zur Wahl. Obwohl der Hickmankatheter mit dem extrakorporalen Zugang eine möglicherweise negative Auswirkung auf die Lebensqualität hat, ist er in Puncto Thrombosierung und Infektanfälligkeit dem Port überlegen.

Operativ stehen sowohl endoskopische, perkutane, als auch offenchirurgische Optionen zur Wahl, welche je nach Fistellokalisation zum Einsatz kommen können.

Endoskopisch kann mittels Clipapplikation oder Endoloop (Ethicon Inc., Somerville, NJ) das endoluminale Ostium verschlossen werden und somit die Fistel zur Abheilung gebracht werden.

Das perkutane Einbringen von Fibrinkleber oder sonstigen Füll- und Klebstoffen kann in Einzelfällen auch in Erwägung gezogen werden, wobei es diesbezüglich nur wenig Erfahrungswerte gibt. Der Vorteil dieser Methode liegt darin, dass bei fehlendem Erfolg die Prozedur komplikationsarm wiederholt werden kann.

Eine definitive operative Sanierung der Fistel sollte in der Regel erst nach 4-6 Monaten in Angriff genommen werden. Manche Autoren propagieren 12-36 Monate zuzuwarten, doch das kumulativ höhere Risiko für wiederkehrende Katheterinfektionen, Leberschäden und womöglich höheres Rezidivrisiko für die Fistel sprechen eher für die kürzere Wartezeit.

Grundsätzlich sollte bei der Operation das gesamte Darmpaket mobilisiert werden und jedwede Verletzungen am Darm weitestgehend vermieden werden. Wenn das Fistelsegment vollständig mobilisiert und dargestellt ist, so ist eine Segmentre- 
sektion des fisteltragenden Segmentes einer Übernähung vorzuziehen. Zur Reanastomosierung stellt die handgenähte Anastomose die bessere Option gegenüber der Stapleranastomose dar. Grund hierfür ist die in diesen Fällen seltener beobachteten Insuffizienzen oder Anastomosenblutungen.

Der Bauchdeckenverschluss ist nicht selten erschwert. Dabei ist eine Mesh-Einlage aufgrund des kontaminierten Operationsgebietes komplikationsträchtig. Die Verwendung von biologischem Netzmaterial, resorbierbaren Netzen oder Einbringung von gestielten Lappen wie z. B. eines Latissimus dorsi flap kann notwendig sein.

In Einzelfällen können Darmverlängerungsverfahren wie das Bianchi und STEP (Serial Transverse Enteroplasty)-Verfahren zum Einsatz kommen. Voraussetzung hierfür ist jedoch das Vorhandensein von dilatierten Darmschlingen als Ausdruck einer vollendeten Adaptation.

Nach scheitern aller o.g. konservativen Therapiemodalitäten kann eine Darmtransplantation als ultima ratio erwogen werden. Kriterien für die gescheiterte Therapie sind:

- zentrale Venenthrombosen mit Verlust von > 2 Zugängen

- eine oder rezidivierende systemische Kathetersepsis mit stationärer Therapie

- katheterassoziierte Pilzinfektion, septischer Schock oder ARDS (acute respiratory distress syndrome)

- rezidivierende Entgleisungen des Flüssigkeits-, Elektrolyt- und Säure/Base-Haushaltes

- fortgeschrittene/irreversible IFALD (Intestinal Failure Associated Liver Disease)

Es können folgende drei Varianten einer Darmtransplantation durchgeführt werden:

- isolierte Darmtransplantation

- kombinierte Leber-Darm-Transplantation

- Multiviszeraltransplantation mit/ohne Leber

Dabei zeigen die Daten ein besseres 5-Jahres-Transplantatüberleben bei der kombinierten Leber-Darm-Transplantation im Vergleich zur isolierten Darmtransplantation ( $46 \%$ vs. 42\%). Grund hierfür scheint eine immunologische Schutzwirkung der Leber auf das Darmpaket zu sein. Im Kontrast dazu steht ein 1-Jahres-Überleben bei Kurzdarmsyndrom mit irreversibler Leberschädigung von 20-30 \%.

\subsection{Komplikationen}

Die Komplikationen können entweder durch das Vorhandensein der Fisteln oder durch die Therapie entstehen. Häufige Komplikationen entstehen durch Sepsis oder Flüssigkeits- und Elektrolytentgleisungen. Dabei stellt eine Sepsis mit ca. 30\% der Fälle die häufigste Todesursache dar. Grund hierfür können intraabdominelle Abs- 
zesse oder katheterassoziierte Infekte (50\%) sein. Die Therapie hierfür sind gezielte Drainagen und eine entsprechende antibiotische oder antimykotische Therapie.

Die langfristige parenterale Ernährung allein hat bereits eine erhebliche Morbidität und eine Mortalität von bis zu 30 \% zur Folge. Das Auftreten von Gallensteinleiden kann gehäuft beobachtet werden, sowie eine progrediente Steatose, Fibrose bis hin zur Zirrhose der Leber (sog. IFALD, Intestinal Failure Associated Liver Disease). 15\% der parenteral ernährten Patienten sind nach einem Jahr betroffen. Die Mortalität hiervon beträgt 100 \% nach zwei Jahren! Es wird an der Optimierung der Zusammensetzung der parenteralen Nahrung gearbeitet. Nach den Empfehlungen der aktuellen S3-Leitlinie sowie der ESPEN scheinen folgende Zusammensetzungen vor Leberschäden zu schützen:

- Proteingehalt $1,5 \mathrm{~g} / \mathrm{Kg} / \mathrm{KG}$

- Glukose und Fett im Verhältnis 60:40

- Omega 3 und Omega 6 Fettsäuren im Verhältnis von 1:1-1:3

Katheterinfektionen stellen eine erhebliche Belastung dar. Autoren gehen von mindestens einem Krankenhausaufenthalt pro Jahr und Patient allein aufgrund einer Katheterinfektion aus. Eine adäquate Pflege des zentralvenösen Zuganges stellt dabei das wichtigste Präventionskriterium dar. Nichtsdestotrotz besteht insbesondere bei Patienten mit Darmfisteln ein besonders hohes Risiko. Das Blocken des Katheters mit Taurolidin hat in einzelnen Studien eine Verringerung der Katheterinfektionen zeigen können.

Aufgrund der oftmals massiven Bauchdeckendefekte und subsequenten Rekonstruktionen sind die Patienten postoperativ besonders gefährdet für Wundinfektionen und Wundheilungsstörungen. Die Wundkomplikationsrate liegt bei $25-50 \%$ und ist bei adipösen Patienten höher. Das Risiko früher Narbenhernien liegt bei 20-30 \%.

Insgesamt besteht trotz maximaler konservativer und operativer Therapie ein Rezidivrisiko von 19\%. Die Gesamtmortalität beträgt 3\% und die Verschlussrate liegt in der aktuellen Literatur bei 80-97 \%. Dabei ist in ca. 30 \% aller Fälle eine konservative Therapie allein ausreichend. Eine Rückkehr zur vollständigen enteralen Ernährung konnte in $79-100 \%$ der publizierten Studien erlangt werden.

\subsection{Prävention}

Die Prävention von Kurzdarmsyndrom und abdominellen Fisteln bedeutet angesichts der Ätiologien zum einen eine aggressive und proaktive Therapie bei Morbus Crohn, zum anderen eine wirkungsvolle Prävention von chirurgischen Komplikationen oder Einschränkung der Darmresektionen. Dies kann zum Beispiel durch zügige Diagnostizierung von Darmischämien und rasche Etablierung von angiographischen Interventionen erfolgen. Im Zweifel kann ein geplanter Second-look zur Vermeidung 
überflüssiger Resektion führen. Bei stenosierendem Morbus Crohn sollte im Zweifel eine Strikturoplastie einer Resektion vorgezogen werden.

Angesichts der Komplikationen unter einer laufenden Therapie eines Darmversagens und/oder kompliziertem Fistelleiden, kann mittels engmaschiger interdisziplinärer Zusammenarbeit und auch mittels Patientenschulung eine wirkungsvolle Reduktion von Infekten oder nutritionellen Dysbalancen erzielt werden.

\section{Weiterführende Literatur}

Bianchi A. Intestinal loop lengthening - a technique for increasing small intestinal length. J Pediatr Surg. 1980;15(2):145-151.

Bharadwaj S, Tandon P, Gohel TD, et al. Current status of intestinal and multivisceral transplantation. Gastroenterol Rep (Oxf). 2017;5(1):20-28.

Grant D, Abu-Elmagd K, Reyes J, et al. Intestinal transplant registry report: global activity and trends. Am J Transplant. 2015;15(1):210-219.

Kim HB, Fauza D, Garza J, et al. Serial transverse enteroplasty (STEP): a novel bowel lengthening procedure. J Pediatr Surg. 2003;38(3):425-429.

Lamprecht G, Pape UF, Witte M, Pascher A. S3-Leitlinie der Deutschen Gesellschaft für Ernährungsmedizin e. V. in Zusammenarbeit mit der AKE, der GESKES und der DGVS. Aktuelle Ernährungsmedizin. 2014;39(2):e57-e71.

Pironi L, Arends J, Bozzetti F, et al. ESPEN guidelines on chronic intestinal failure in adults. Clin Nutr. 2016;35(2):247-307.

Rege A. The Surgical Approach to Short Bowel Syndrome - Autologous Reconstruction versus Transplantation. Viszeralmedizin. 2014;30(3):179-189.

Schaffler H, Daraban AF, Roggenbrod S, et al. Characterization of refractory port-related blood stream infections in intestinal failure patients on parenteral nutrition. Z Gastroenterol. 2011;49(3):335-339.

Thompson JS. Short Bowel Syndrome and Malabsorption - Causes and Prevention. Viszeralmedizin. 2014;4(30):174-178.

de Vries FEE, Atema J, van Ruler 0, et al. A Systematic Review and Meta-analysis of Timing and Outcome of Intestinal Failure Surgery in Patients with Enteric Fistula. World J Surg. 2018;42(3):695-706.

Williams LJ, Zolfaghari S, Boushey R. Complications of Enterocutaneous Fistulas and Their Management. Clin Colon Rectal Surg. 2010;23:209-220.

www.espen.org

www.dgem.de 
